\title{
El Sector Construcción: la problemática nacional y la importancia de la normativa técnica como ejes en la solución de problemas
}

\author{
The Construction Sector: national issues \\ and the importance of technical standards \\ as axes in troubleshooting
}

Adelina Ortega-Rojas'

Fecha de recepción: 12 de mayo de 2014

Fecha de aceptación: I I de setiembre de 2014

Ortega-Rojas, A. El Sector Construcción: la problemática nacional y la importancia de la normativa técnica como ejes en la solución de problemas. . Tecnología en Marcha. Edición especial Movilidad Estudiantil 20I4. Pág. 58-67. 


\section{Palabras clave}

Construcción; Costa Rica; normativa; OSHA; reglamentación; seguridad laboral; vivienda.

\section{Resumen}

El abordaje de la situación del Sector Construcción en Costa Rica puede decirse que tiene aspectos multifactoriales que inciden directamente en la forma en que este sector se ha venido desarrollando en los últimos años, como coadyuvante de la dinámica en la economía del país.

En el sentido señalado, vale anotar que el Sector Construcción en los últimos años ha tenido un comportamiento creciente, a excepción del año 2013 cuando sufrió una baja significativa; no obstante, entidades entendidas en la materia como la Cámara Costarricense de construcción (CCC) son optimistas en la estabilidad en términos generales de este sector, referente específicamente en un primer orden a la construcción de vivienda.

En el contexto indicado, reviste singular importancia la consideración de la normativa referente a aspectos de seguridad laboral que rige el Sector Construcción en nuestro país, normativa tanto nacional como de índole internacional, que ha venido a buscar reglamentar y apoyar desde la perspectiva de disminución de los riesgos laborales, lo que viene preocupando a entidades gubernamentales como el Instituto Nacional de Seguros, que ha hecho esfuerzos al respecto.

Desde esta perspectiva, el Sector Construcción se ha venido dinamizando tanto desde la perspectiva económica y de impacto para el país, como en la necesidad de retomar controles y normativas que venga a dar consistencia a este sector, no solo en el ámbito económico señalado sino desde la óptica de la seguridad laboral, que ha estado realizando propuestas importantes y necesarias para la estabilidad de este sector.

\section{Keywords}

Construction; Costa Rica; legislation; OSHA; regulation; Occupational Safety; Housing.

\begin{abstract}
The approach to the situation of the Construction Sector in Costa Rica can be said to have multifactorial issues that directly affect the way in which this sector has been developing in recent years, as an adjunct to the dynamics of the economy.
\end{abstract}

In the sense above, is worth noting that the construction sector in recent years has had an upward trend except in 2013 when it suffered a significant decrease, however relevant entities on the field as the Costa Rican Chamber of Construction (CCC) are optimistic overall in the stability of this sector, specifically in at first to the housing construction.

In the context above, is of particular importance the consideration of the rules regarding aspects of occupational safety that governs the Construction Sector in our country, both national legislation and international, which has come to look regulate and support from the perspective of reducing occupational risks, and they are of concern to governmental entities such as the National Insurance Institute, which has made efforts in this area.

From this perspective, the construction sector has been invigorating both from an economic perspective and impact for the country and the need to resume controls and regulations that come to give consistency to this sector, not only in the economic field, but in the occupational safety, which has been making important and necessary proposals for the stability of this sector. 


\section{Introducción}

La presentación que aquí se realiza pretende dar a conocer en primera instancia la situación actual del Sector Construcción en Costa Rica, donde se esboza no solo la realidad, sino situaciones que atañen como dificultades para este sector. Por otra parte, se introducen datos estadísticos que señalan el comportamiento respecto al crecimiento y decrecimiento del mencionado sector, y que debe señalarse como punto importante la afectación de la crisis mundial a este sector, sin dejar de lado y como ejemplo la tasa de accidentes señalada por el Instituto Nacional de Seguros (INS), que se vincula de manera importante con las normas propias de salud ocupacional con que cuenta el país.

De igual forma, se presenta la normativa que tanto a nivel internacional como nacional cubre el sector de la economía mencionado, señalándose de manera medular las consecuencias de la aplicación de dicha normativa y el impacto a nivel de dicho sector, con lo que se elaboran conclusiones relacionadas con el punto antes indicado.

\section{El sector construcción en Costa Rica, impacto y cobertura}

La importancia del Sector Construcción en la economía costarricense, de acuerdo con la Cámara
Costarricense de Construcción (CCC), se ve reflejada en el aporte que da al crecimiento de la producción nacional, generación de empleo de manera inmediata y de muchos sectores productivos proveedores de esto. Así, esta industria toma un papel principal en los procesos de crecimiento y desarrollo nacional (CCC, 20I3).

En este sentido, en Costa Rica la industria de la construcción posee un carácter dinámico, debido en gran medida a la fuerte correlación con otras variables macroeconómicas y con el desempeño de la economía nacional, por lo que su evolución dependerá del comportamiento de la actividad económica en general. Esto se evidencia en la figura I, que está basado en datos de los Informes Económicos del Sector Construcción generados por la Cámara Costarricense de Construcción, donde, según estos informes, durante el año 2012 el Sector Construcción enfrentó un panorama alentador, donde la recuperación del mismo, se vislumbró cada vez mejor; sin embargo, en el año 2013 la situación fue contraria donde los indicadores mostraron situaciones de desaceleración y hasta decrecimiento (CCC, 2013).

No obstante, según los informes de la CCC mencionados anteriormente, en el sector privado, la construcción de nuevas edificaciones depende del comportamiento de los precios de los insumos utilizados, de las tasas de interés, así como del acceso

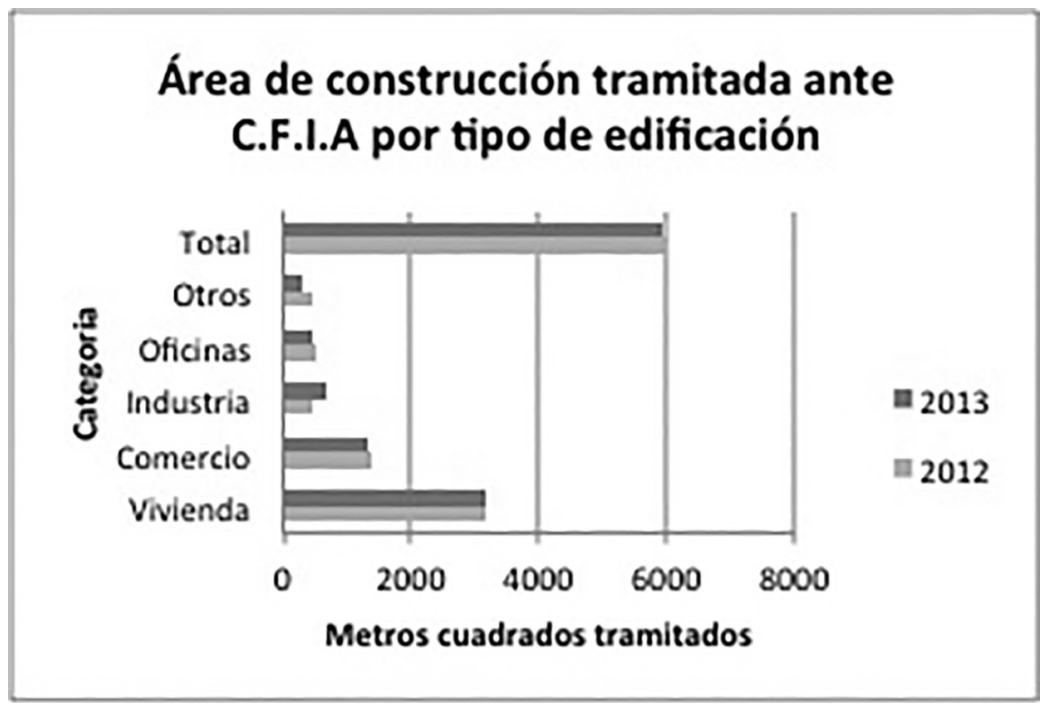

Figura I. Área de construcción tramitada ante C.F.I.A por tipo de edificación 20I2-20I3. 
al crédito. Pero, del lado de la infraestructura pública, la construcción depende tanto del nivel de ingresos del Estado, como de la decisión de invertir en nuevas edificaciones; es decir, que el sector construcción a la vez que afecta la economía, es afectado por ella, ya sea en el ámbito privado o público.

Por ello, tal y como lo muestran los datos del Colegio Federado de Ingenieros y Arquitectos (CFIA), en nuestro país la mayor cantidad de metros cuadrados tramitados para construcción en los últimos años fue para el sector vivienda, como se observa en la figura I.

De igual forma, en la figura I se observa que para el año 2013, se tramitaron para efectos de construcción menos metros cuadrados que en el año 2012. Según datos del Informe Económico del Sector Constructor de diciembre 20 I 3, exactamente 99.000 metros cuadrados menos de construcción. Asimismo, se recalca que siempre la categoría de vivienda se coloca en primer lugar a pesar de la disminución de metros cuadrados tramitados de un año al otro (CCC, 20 I3).

En los países en vía de desarrollo, la construcción de viviendas es de suma importancia debido a la elevada tasa de crecimiento de población, lo cual se ilustra con el figura 2, donde se muestra la población total por año de algunos países latinoamericanos en vías de desarrollo, dentro de los cuales está Costa Rica. En el caso particular de este país, el crecimiento habitacional ha colocado a la vivienda en un lugar prioritario de la agenda nacional.

De lo anterior, se deduce que las entidades competentes (CCC y CFIA) han sido categóricas en que la construcción de vivienda se ha mantenido como un sector creciente, tanto en la agenda nacional de la empresa pública y privada como de la economía nacional.

\section{Normativa aplicable para el Sector de Construcción en Costa Rica}

Dada la significancia y la importancia de este sector en la economía nacional, en la agenda gubernamental, para el ámbito público y privado, es un sector que no está ajeno a la normativa que le cubre. Es así como conviene señalar que existe normativa internacional como la normativa estadounidense de la Administración de Seguridad y Salud Ocupacional (OSHA), además de las leyes, reglamentos propios y obligatorios para el sector de construcción tales como el Reglamento General de Salud Ocupacional del Ministerio de Trabajo y Seguridad Social, Reglamento del CFIA, Reglamentos del Ministerio de Salud, tales como permisos de funcionamiento, plan de salud ocupacional, plan de emergencias, plan de manejo de desechos, entre otros. También existen otras nacionales que son de interés particular y opcionales, en el tanto se quiera dar valor agregado al proyecto o entidad, tal como las normas propias

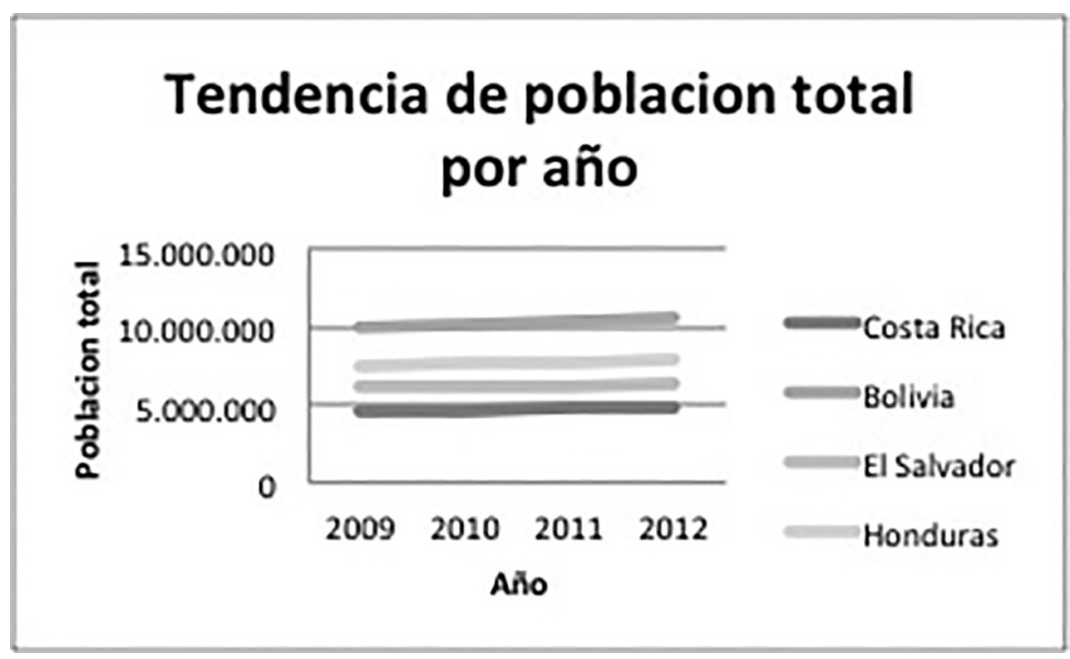

Figura 2. Población total por año en países en vía de desarrollo.

Fuente: Elaboración propia de la estudiante, con base en Indicadores del desarrollo mundial del Banco Mundial, 20 I4. 

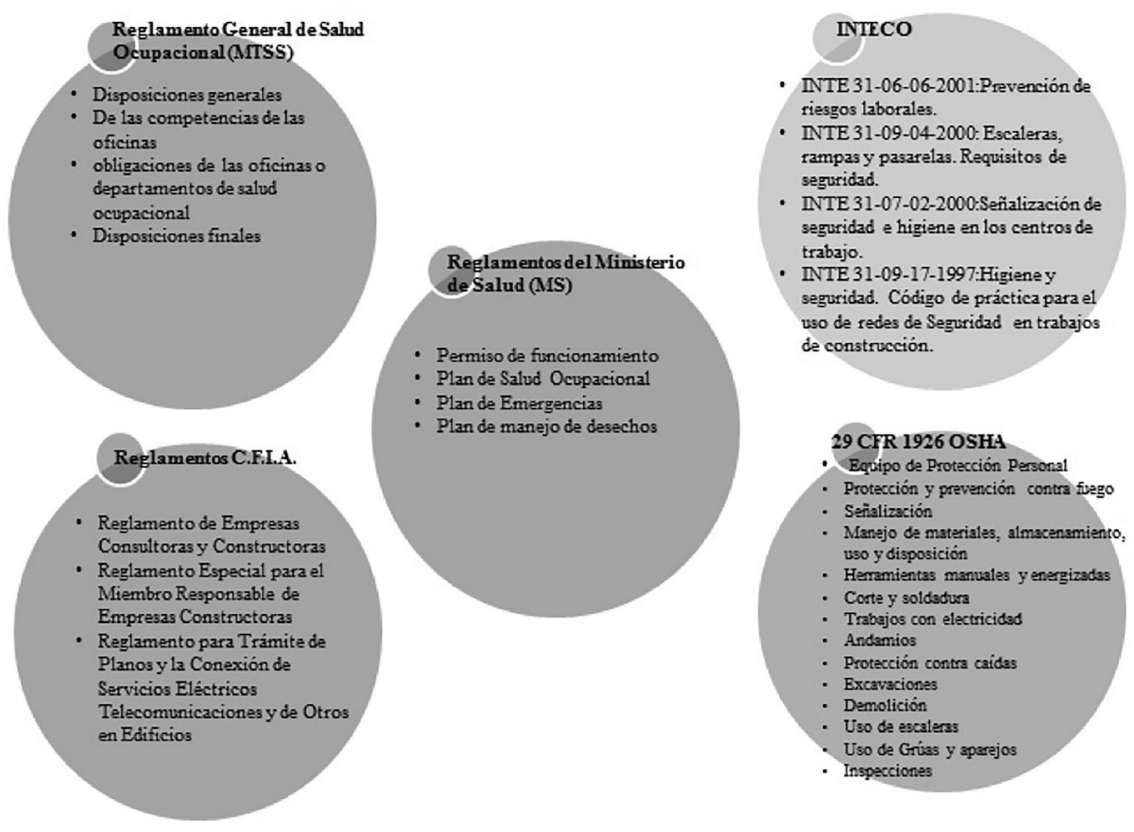

Figura 3. Reglamentos y normativas nacionales e internacionales.

Fuente: elaboración propia, 2014.

del Instituto de Normas Técnicas de Costa Rica (INTECO).

La figura 3, pretende visualizar lo antes indicado, pudiéndose observar tanto normativa de orden internacional como códigos, reglamentos y disposiciones nacionales que atañen directamente para cumplir con el sector de construcción.

Se puede concluir de la figura 3, que la normativa referente a seguridad en materia de construcción en Costa Rica es abundante, las principales entidades reguladoras del ámbito laboral y, sobre todo, de la construcción (MTSS, CCC, MS), cuentan con regulaciones que confieren dicho tema.

Sin embargo, la existencia de esta cantidad de reglamentos no implica que existan y se apliquen los controles adecuados para regularlos y velar por su cumplimiento, ni que dichas regulaciones sean lo suficientemente detalladas para la comprensión de la población del Sector Construcción.

Por otro lado, OSHA en su regulación 29 CFR 1926, Regulaciones para la industria de la Construcción, detalla minuciosamente e ilustra con ejemplos claros situaciones críticas que se pueden presentar comúnmente. Además, OSHA cuenta con controles ya estipulados por la mencionada entidad y el Departamento Laboral de Estados Unidos indica que de no cumplirse pueden incurrir en sanciones económicas, procesos de inspección y hasta el cierre de las obras.

Contrariamente, la vinculación de los reglamentos y los debidos controles que faciliten el velar por el cumplimiento de estos es primordial para lograr un eficiente de la regulación vigente.

\section{Situación actual del Sector Construcción en Costa Rica}

Según el último dato reportado por el Instituto Nacional de Seguros (INS), en el año 2006, respecto a la alta incidencia y severidad de accidentes, el sector construcción ocupó en Costa Rica el segundo lugar en el índice de incidencia y en el de gravedad. La cobertura del régimen de riesgos del trabajo, para ese año, fue de un $74 \%$, con respecto a la población asalariada ocupada(Hernández, Arias, Rodríguez, Araya, Mata y Medina, 20I2).

En este contexto, el Instituto Nacional de Seguros registró en el 2006 un total de 78.300 trabajadores 


\section{Poblacion ocupada en el Sector Cosntrucción por año}

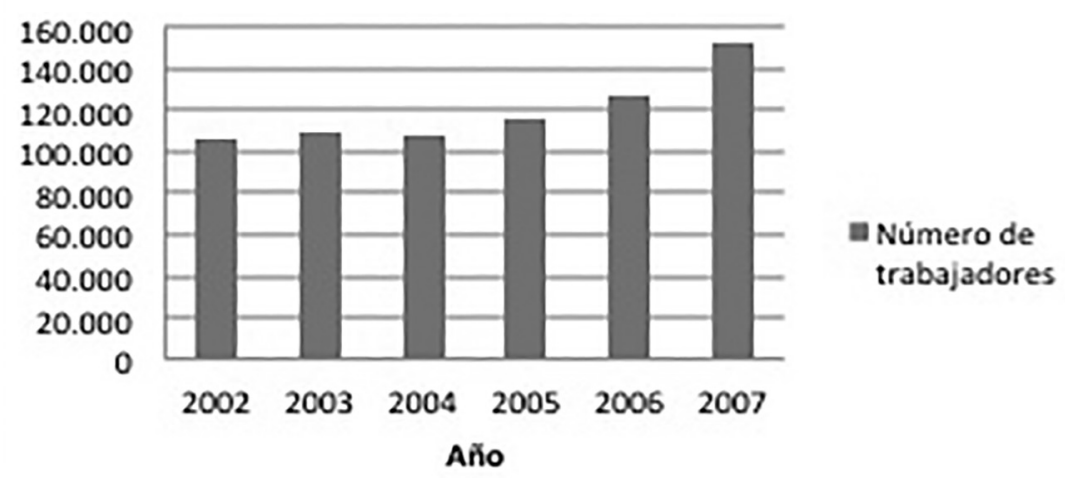

Figura 4. Población ocupada en el ámbito de la construcción en Costa Rica, años 2002-2007.

Fuente: Gómez, 2008.

asegurados bajo la Póliza de Riesgos de Trabajo. Durante este periodo, se registraron 18528 accidentes, para un total de 28665 I días de incapacidad. Siendo la mayoría de estos accidentes producidos por faltas a la normativa de salud ocupacional, aspectos de seguridad deficientes (Hernández et al, 20 I2).

En la figura 4, que se indica a continuación, se destaca el número de personas trabajadoras que laboraron en el sector construcción del año 2002 al 2007, datos obtenidos por el Instituto Nacional de Estadística y Censos (INEC) en la Encuesta de Hogares de propósitos múltiples.

Según la Encuesta Nacional de Hogares, elaborada por el INEC, para julio del 2013 se contabilizaron I |4.639 trabajadores ocupados en el sector construcción. Para el 2013, se generaron, en promedio, cerca de 80500 empleos formales en el sector construcción, lo que representa un crecimiento de 0,7\% (600 empleos formales más), comparando contra el 2012 (CCC, 20I3). Estas cifras tienen que ver con el impacto sobre el país de la crisis mundial y que entre otros sectores, afectó de manera cierta el Sector Construcción.

La EncuestaTrimestral sobre Opinión de Empresarios (ETOE), efectuada por el Instituto de Investigaciones en Ciencias Económicas (I.I.C.E.) de la Universidad de Costa Rica, correspondiente al IV Trimestre 2013 , indicó que el $62 \%$ de las empresas del sector construcción consultadas, reportó que esperaba mantener el nivel de contratación del III Trimestre 20 I3. Mientras tanto, un 34\% indicó que aumentaría el nivel de contratación en el IV trimestre del 20/3, aduciendo que durante estos meses se incrementa el nivel de trabajo. Finalmente, el $4 \%$ restante de los empresarios afirmó que más bien durante el IV trimestre 2013 reduciría su planilla (CCC, 20I3).

Lo descrito permite deliberar que al mantenerse - aumentar el número de trabajadores en el mencionado sector, el número de accidentes en este sector laboral también deberá aumentar, lo que incidirá en el uso de la normativa propia de salud ocupacional.

Según un estudio realizado por el Instituto Nacional de Aprendizaje (INA), el promedio del nivel de escolaridad de los trabajadores del sector construcción, es primaria completa para maestros de obra, carpinteros, albañiles, fontaneros y soldadores. Solamente, los electricistas cuentan con secundaria completa. Por ello se concluye que niveles altos de escolaridad no son requisitos básicos para la contratación de recurso humano en las empresas del Sector Construcción (Gómez, 2008). 
Los trabajadores sin capacitación técnica u otra formación no poseen ningún tipo de capacitación formal en materia de seguridad para realizar los trabajos de construcción debidamente, y en la mayoría de casos las empresas constructoras no cuentan con personas capacitadas en esta área para que ofrezcan la capacitación requerida.

En virtud de lo indicado, Costa Rica está rezagada en lo que respecta a regulación e inspecciones gubernamentales en materia de seguridad laboral, lo que es una necesidad sobre todo en el Sector Construcción, debido a la cantidad de riesgos a los que se exponen los trabajadores.

La Administración de Seguridad y Salud Ocupacional (OSHA) de Estados Unidos es un ente creado para asegurar las condiciones de trabajo seguras y saludables para los trabajadores y trabajadoras mediante la creación y aplicación de normas, que procura la capacitación, difusión, educación y asistencia para esta población.

Dentro de las funciones de la citada entidad, ofrece capacitación a los distintos niveles de la organización en las empresas constructoras. En el caso de los cursos para personas encargadas del área se seguridad y salud, el curso \#510, Normas de Seguridad y Salud Ocupacional para la Industria de la Construcción, cubre las normas de seguridad OSHA, las políticas y procedimientos en el sector de la construcción.

Los temas incluyen el alcance y la aplicación de las normas de construcción de OSHA, seguridad en la construcción y los principios de salud, y un énfasis especial en aquellas áreas de la construcción que son consideradas las más peligrosas. Esto como ejemplo de todo lo antes anotado y teniendo como fin que las personas encargadas de la seguridad y salud en las empresas constructoras sean capaces de definir los términos de la construcción que se encuentran en las Normas de Construcción de OSHA, identificar los riesgos que se producen en la industria de la construcción, localizar, determinar y aplicar las normas de construcción apropiadas de OSHA, políticas y procedimientos, y describir el uso de las Normas de Construcción de OSHA y reglamentos para complementar un programa de seguridad y salud.

Capacitación de esta naturaleza son necesarios en Costa Rica, donde se busque capacitar a las personas que trabajan en construcción en materia de seguridad y salud, desde los altos mandos de la empresa, para que conozcan sus responsabilidades como patronos; pasando por los encargados de seguridad para que puedan aplicar la normativas, políticas, procedimientos e instrucciones, tanto nacionales como internacionales, y llevarlo hasta los obreros con el fin de que conozcan sus deberes y derechos en esta materia, así como los riesgos a los que se exponen y puedan proteger su propia salud.

La capacitación es un componente primordial para la realización correcta de cualquier labor del ámbito del trabajo. En el caso particular de los trabajos del sector de la construcción representa una posibilidad para disminuir los riegos que pueden llegar a causar accidentes 0 incidentes laborales, los cuales son comunes en este ámbito laboral, tal y como se ha señalado.

\section{Agradecimientos}

La autora expresa su agradecimiento al "Programa de Pasantía Estudiantil con Fondos del Sistema 20I4, CONARE-TEC" con el cual se ha cubierto parte fundamental de los gastos de viaje del pasante y a la Rectoría del Instituto Tecnológico que gestiona todo el programa.

\section{Conclusiones}

Siendo Costa Rica un país de Derecho, donde las leyes, códigos y reglamentos están presentes en el accionar de los diferentes sectores productivos del país, aunado además por la normativa de orden internacional con las que el país ha entrado en tratados y convenios, el sector construcción no está exento de contar con una serie de normativas, tal y como anteriormente se indicó.

No obstante, adolece el país de una aplicación sistemática y vinculada, a como lo señala la norma, al contrario y tal y como se enunció en las estadísticas arriba indicadas el desfase entre la propuesta y la aplicación es lo que produce dichas estadísticas; por ejemplo, respecto a accidentes laborales.

En virtud de lo aquí señalado, y como corolario se debe tener claridad que lo que se requiere es una aplicación eficiente de toda la normativa de Salud Ocupacional que rige el país, lo que evitaría los riesgos 
a los que está expuesta la población trabajadora del sector construcción y que, obviamente, se debe ser rígido y exigente en la aplicación de la consabida normativa.

\section{Bibliografía}

Cámara Costarricense de Construcción (2013). Informe económico del sector construcción (No. 12) San José: Cámara Costarricense de Construcción.

Cámara Costarricense de la Construcción (s.f.). Http://www. estadonacion.or.cr/files/biblioteca_virtual/0 I/Sectorconstruccion.pdf (No. 13) San José: Cámara Costarricense de la Construcción.

Colegio Federado de Ingenieros y Arquitectos de Costa Rica (20|4). Trámites y servicios leyes y reglamentos Recuperado desde: http://www.cfia.or.cr/leyes.htm

Gómez, M. (2008). Estudio de determinación de necesidades de capacitación y formación profesional en el sector de la construcción a nivel nacional. Recuperado desde: http://www. ina.ac.cr/institucional/institucional/construccion.html
Grupo del Banco Mundial (20/4). Población, total Recuperado desde: http://datos.bancomundial.org/indicador/SP.POP. TOTL/countries?display=graph

Hernández, M., Arias, E., Rodríguez, M., Araya, T., Mata, C. \& Medina, L. (20/2). Mejoramiento de las Condiciones de Seguridad e Higiene Laboral en el sector construcción en Costa Rica Recuperado desde: http://bibliodigital.itcr.ac.cr/ xmlui/bitstream/handle/2238/3 |44/mejoramiento_condiciones_seguridad_higiene_laboral.pdf?sequence $=$ |

Ministerio de Trabajo y Seguridad Social (1998, 25 de mayo). Reglamento sobre las oficinas o departamentos de salud ocupacional Recuperado desde: http://portal.ccss. sa.cr/portal/page/portal/Gerencia_Medica/DSS_Central_ Sur/SaludOcupacional/ReglamentoDeptoSaludOcupacional. pdf

Occupational Safety and Health Administration (2013). 29 CRF 1926 OSHA, Construction Industry Regulations (2013 ed.) lowa: Mancomm.

United States Department of Labor (20|4). Course Descriptions Recuperado desde: https://www.osha.gov/dte/ edcenters/course_description.html 